\title{
A PRODUÇÃO ACADÊMICA SOBRE ESTRATÉGIAS E/DE INOVAÇÃOEM ENGENHARIA DE PRODUÇÃO
}

\author{
Claudio Sonaglio Albano \\ Caio Marcello Recart da Silveira \\ Andressa de Oliveira Rodrigues \\ Wanderson Lombardy Pereira \\ claudio.albano@gmail.com \\ caio.silveira@unipampa.edu.br \\ andressa.or@hotmail.com \\ wandersonlombardy@hotmail.com \\ Universidade Federal do Pampa - UNIPAMPA
}

\begin{abstract}
Resumo
O trabalho aborda os temas estratégia e inovação, relativamente à produção científica, no âmbito de trabalhos publicados na base de dados da Coordenação de Aperfeiçoamento de Pessoal de Nível Superior (CAPES) na área da Engenharia de Produção. Por intermédio de questões relacionadas à identificação dos autores mais produtivos, suas instituições de origem, os ambientes de pesquisa onde os estudos foram desenvolvidos e os periódicos onde os trabalhos foram publicados foi possível elaborar um diagnóstico (perfil) e o atendimento dos objetivos propostos e que estão relacionados com as questões norteadoras. Foram consultadas bases de periódicos pela classificação Qualis, sendo o recorte orientado pela classificação A1, A2, B1 e B2, na área de Engenharia da Produção (Engenharias III). Chama atenção a ausência de organizações públicas nos trabalhos, este fato talvez mereça uma maior investigação dos motivos que fazem com que as organizações públicas não sejam objeto de estudos relacionados a estratégia, mas especialmente relacionados a inovação.
\end{abstract}

Palavras-chave: Estratégias e/de Inovação. Produção Acadêmica. Engenharia de Produção.

\begin{abstract}
.
The work addresses the themes and innovation strategy with regard to scientific production, as part of works published in the database of Higher Education Personnel Improvement Coordination (CAPES) in the area of Production Engineering. Through issues related to the identification of the most productive authors, their home institutions, research environments where the studies were developed and journals where the papers were published it was possible to make a diagnosis (profile) and meeting the proposed objectives and they are related to the guiding questions. Periodicals for Qualis classification bases were consulted, and the cut-driven classification A1, A2, B1 and B2, the Production Engineering Department (Engineering III). Noteworthy is the absence of public organizations in the work, this fact may deserve further investigation of the reasons that make the public organizations are not subject to studies related to strategy, but especially related to innovation.
\end{abstract}


Keywords: Strategies and/to Innovation. Academic Production.Production Engineering.

\section{Introdução}

Este trabalho aborda a temática de estratégias e/de inovação, referentes à produção científica, no âmbito de trabalhos publicados em revistas na basede dados da Coordenação de Aperfeiçoamento de Pessoal de Nível Superior (CAPES). Justifica-se a escolha de revistas da CAPES, pois no contexto brasileiro esta base de dados analisa a produção intelectual por intermédio do 'processo de atualização' da classificação de periódicos.

A motivação para este trabalho está orientada pela necessidade de conhecer, o que tem sido produzido no Brasil, em relação à temática de estratégias e/de inovação. Este fato decorre da perspectiva de que as instituições de ensino superior desempenham um papel preponderante nas relações sociais por intermédio da produção de conhecimento.Desta forma uma pesquisa sobre abrangência das pesquisas bibliométricas revela que muitos trabalhos foram desenvolvidos nas últimas décadas no contexto brasileiro.

Além dos fatos citados no parágrafo anterior, as questões relacionadas com as estratégias e/de inovação permeiam diferentes contextos organizacionais em virtude da sua importância decorrente da estreita vinculação com produtos, processos e pessoas no âmbito das organizações.

Os autores desse trabalho, visando identificar trabalhos bibliométricos, encontraram estudos nas mais diferentes áreas. Foram encontrados estudos sobre Engenharia de Produção (BERTO E NAKANO, 2000; AMARAL ET AL., 2004; SANTOS, PILATTI E KOVALESKI, 2005; LACERDA ET AL., 2007). Destaca-se que outros estudos, em diferentes áreas, foram encontrados (HOPPEN, 1998; HOPPEN E MEIRELLES, 2005; LOIOLA E BASTOS, 2003; TONELLIET AL., 2003; DUARTE, PINTO E OLIVEIRA FILHO, 2004; CALDAS, 2005; TINOCO, 2005; CALLADO E ALMEIDA, 2005; JABBOUR, SANTOS E BARIBIERI, 2008; GOMES, MACHADO E GIOTTO,, 2009; ROSSONI, FERREIRA JÚNIOR E HOCAYEN-DASILVA, 2009; MARCANTONIO E LAZZERI, 2014). Nestes estudos não foram encontrados estudos bibliométricos sobre estratégias e/de inovação.

Em relação à singularidade, esta se prende ao interesse em verificar o que tem sido produzido no Brasil, no espaço representado pelos periódicos nacionais consultados, através da produção de trabalhos relativamente ao tema das estratégias e/de inovação, bem como as indagações associadas e listadas como questões norteadoras.Em decorrência das diferentes possibilidades de acesso as bases de dados, reconhecemos a possibilidade de ausência de algum estudo que trate sobre o tema estratégias e/de inovação.

Assim, pretende-se responder às seguintes questões norteadoras da pesquisa sobre estratégias e/de inovação: Quais os autores que publicam trabalhos relacionados a estratégia elde inovação? Qual a origem institucional dos autores citados? Quais os ambientes setoriais, multisetoriais e espaciais onde os trabalhos foram desenvolvidos? Quais os periódicos em que estes trabalhos consultados foram publicados?

Com base nestas questões, pretende-se apresentar um diagnóstico sobre os trabalhos desenvolvidos no Brasil e relacionados com o tema das estratégias e/de inovação. Ao final deste estudo espera-se contribuir com a área ao apresentar um panorama da produção científica junto às bases de dados dos periódicos selecionados e consultados.

O trabalho está estruturado em cinco seções representadas por esta introdução, logo após, na seção dois, a fundamentação teórica. Na terceira seção, são apresentados os procedimentos 
metodológicos, os resultados compõem a quarta seção e finalmente a quinta seção apresenta as considerações finais, limitações do trabalho e sugestões para futuros trabalhos.

\section{Fundamentação teórica}

Esta seção pretende discutir, conceitos relacionados com as temáticas inovação, estratégia e bibliometria. A temática da inovação discutida em diferentes trabalhos (Neely e Hii, 1998; Cassiolato e Lastres, 2000; Calligaris et al., 2001; Barbieri, 2003; Mowery e Rosenberg, 2005; Kim e Nelson, 2005; Nelson, 2006; Freeman e Soete, 2008) permeia todo e qualquer contexto organizacional em virtude da sua vinculação com produtos, processos e pessoas e o dinamismo relacionado com a produtividade e a competitividade das firmas.

A inovação (Manual de Oslo, 2005; Pesquisa de Inovação Tecnológica (PINTEC), 2005; Lei 10.973; Decretolei 5.563; Decreto 5.798; Lei 13.243)se configura pela implementação de um produto novo ou aperfeiçoado, de um processo, de um método de marketing ou organizacional novos, na organização do trabalho ou nas relações entre as empresas e o entorno. Isto se aplica tanto no caso em que a empresa é a pioneira, quanto na adoção de desenvolvimentos de outras empresas.

A inovação pode se apresentar como uma atividade precursora nascida das competências internas da empresa (Kim e Nelson, 2005, p. 16), se caracterizar por um determinado estado de descobrimento de idéias e mudanças (Neely e Hii, 1998, p. 8), pelo domínio de conhecimentos relacionados com projetos de produtos e processos (Nelson, 2006, p. 430) e pela perspectiva de mudança compartilhada pelo crescimento econômico, pela qualidade de vida, a conservação dos recursos naturais e a melhoria do meio ambiente (FREEMAN E SOETE, 2008, p. 19). A inovação é definida no Manual de Oslo (Ministério da Ciência e Tecnologia, 2005) comoa implementação de um bem ou serviço novo ou significativamente melhorado, ou um processo, ou um novo método de marketing, ou um novo método organizacional nas práticas de negócios, na organização do local de trabalho ou nas relações externas.

Em relação às atividades de inovação, o Manual de Oslo (Ministério da Ciência e Tecnologia, 2005, p. 56) caracteriza-as como as 'etapas científicas, tecnológicas, organizacionais, financeiras e comerciais que conduzem, ou visam conduzir, à implementação de inovações'. Depreende-se que uma inovação para configurar-se deve ser implementada, ou seja, introduzida no mercado.

Com relação ao termo estratégia, não existem mais dúvidas sobre sua importância para o planejamento de uma organização, visando sua introdução ou sobrevivência em seu mercado competitivo. Segundo Mintzberget. al. (2000) a definição de uma estratégia, não garante a previsão e resolução de todos os problemas, entretanto ajuda ao oferecer suporte às ações que podem mitigar estes problemas.

O conceito de estratégia nasceu da necessidade de realizar objetivos em situações de concorrência, como no caso da guerra, nos jogos e nos negócios. Na academia em meados da década de 50, registram-se os primeiros estudos sobre estratégia. Existem diversas conceituações para estratégia, segundo Hitt \&Ireland (2007) estratégia é um conjunto integrado e coordenado de compromissos e ações definidos para explorar competências essenciais e obter vantagem competitiva.

Com relação ao termo estratégia, não existem mais dúvidas sobre sua importância para o planejamento de uma organização, visando sua introdução ou sobrevivência em seu mercado competitivo. Existem diversas definições para este termo, mas segundo Mintzberg et. al., (2000) existe a necessidade de uma definição mais completa de estratégia (a partir dos 5ps), pois como: plano, a estratégia tem por finalidade estabelecer direções para a organização; trama (ploy), a 
estratégia é utilizada para ameaçar e confundir os concorrentes, na busca de vantagens; padrão, a estratégia deve considerar o comportamento e assimilação das ações de sucesso no processo decisório; posição, a estratégia encoraja as organizações a analisarem o ambiente, buscando uma posição que as protejam, visando defender-se e influenciar a competição; e finalmente como perspectiva, a estratégia traz questões referentes à intenção e comportamento em um contexto coletivo. Ainda segundo este autor, o planejamento estratégico, a partir da definição de uma estratégia, não garante a previsão e resolução de todos os problemas, ajuda ao oferecer suporte às ações que podem mitigar estes problemas.

A relação entre inovação e estratégia foi objeto de observação em alguns estudos como o desenvolvido por Yang (2010) onde a autora destaca a importância estratégica da inovação na indústria farmacêutica e destaca que estas estratégias precisam de um tipo de regime corporativo entre equipe de inovação da empresa e outros atores para o seu desenvolvimento. Em Vitorelli (2010) a autora trabalha as temáticas da inovação aberta e o contexto das redes organizacionais como alternativas estratégicas para o processo inovativo e representados por dois estudos de casos realizados em indústrias do setor químico. Estes e outros estudos apresentam as temáticas da estratégia e da inovação como elementos determinantes no processo inovativo das empresas e/ou setores estudados (Gattaz, 2010; Souza, 2010; para mencionar dois destes).

Segundo Hoppen et. al, (1998) e Marcantonio e Lazzeri (2014) a bibliometria é uma área do conhecimento que através de métodos estatísticos, permite analisar e construir indicadores sobre a evolução da produção científica em áreas, organizações e até mesmo países. Tem abrangência interdisciplinar ou multidisciplinar, podendo ser aplicada a diversas áreas do conhecimento, entre seus objetivos pode-se destacar: identificar tendências e crescimento do conhecimento em uma determinada área; identificar autores e instituições mais produtivos; identificar periódicos, revistas ou eventos núcleos de cada área, entre outros.

\section{Procedimentos Metodológicos}

Este estudo, com características exploratórias conforme Gil (2002), foi desenvolvido em um cenário em que encontramos poucos trabalhos conforme apresentado na introdução. Um estudo exploratório é realizado em área que haja pouco conhecimento acumulado e sistematizado.

O trabalho foi realizado através de quatro etapas. Na primeira etapa foi realizada a seleção dos periódicos a serem consultados com base na classificação Qualis Periódicos da CAPES. Na segunda etapa foi desenvolvido um levantamento exploratório, por termos de consulta nos periódicos selecionados com base na consulta mencionada na etapa um, para verificar possíveis trabalhos relacionados com o descritor 'estratégias e/de inovação', entre os anos de 2012 e 2014, verificando a existência deste termo de consulta nas partes representadas pelo título e/ou resumo e/ou palavras-chave dos artigos consultados. Uma vez identificados e selecionados, os mesmos foram catalogados.

A terceira etapa 3 foi caracterizada pela elaboração de fichamentos contendo a referência bibliográfica do estudo consultado e as informações relacionadas com as indagações presentes nas questões de pesquisa. A fase conclusiva constituiu-se das caracterizações relacionadas com as questões de pesquisa supramencionadas e o tratamento analítico-conclusivo destas informações.

Estas bases foram orientadas pela classificação Qualis Periódicos da CAPES dos mesmos e o recorte foi nas bases de dados dos periódicos classificados como A1, A2, B1 e B2, na área de Engenharia da Produção (Engenharias III). Em relação ao método de investigação a pesquisa caracteriza-se por ser bibliográfica (MIGUEL, 2012). 


\section{Resultados}

Os resultados não estão apresentados obedecendo à ordem proposta para as questões norteadoras do trabalho, mas sim visando atender a uma melhor leitura e interpretação dos resultados. Desta forma, no tabela 1, estão relacionados os autores, em ordem alfabética, que publicaram trabalhos relacionados a estratégia e/de inovação e respectivos periódicos.

Tabela 1 - Autores, período publicação e periódicos

\begin{tabular}{|c|c|}
\hline Autores, Ano & Periódico \\
\hline César Augusto Biancolino, 2013 & Revista Brasileira de Gestão de Negócios \\
\hline ClândiaMaffini Gomes, 2014 & Revista Brasileira de Gestão de Negócios \\
\hline Eliete Pozzobon Palma, 2014 & Revista Brasileira de Gestão de Negócios \\
\hline Emerson AntonioMaccari, 2013 & Revista Brasileira de Gestão de Negócios \\
\hline Fábio Luiz ZandovalBonazzi, 2014 & Revista Brasileira de Gestão de Negócios \\
\hline Fernanda Lazzari, 2014 & Produção Online \\
\hline Gabriel Sperandio Milan, 2014 & Produção Online \\
\hline Jonas Mendes Constante, 2014 & Produção Online \\
\hline Jordana Marques Kneipp, 2014 & Revista Brasileira de Gestão de Negócios \\
\hline Julieta Scheidt Dienstmann, 2014 & Produção Online \\
\hline Leonardo Ensslin, 2014 & Produção Online \\
\hline Luciana Aparecida Barbieri da Rosa, 2014 & Revista Brasileira de Gestão de Negócios \\
\hline Manuel Francisco Suárez-Barraza, 2013 & Revista Brasileira de Gestão de Negócios \\
\hline Maurício Fernandes Pereira, 2013 & Revista Brasileira de Gestão de Negócios \\
\hline Moises Ari Zilber, 2014 & Revista Brasileira de Gestão de Negócios \\
\hline Nathalia Fiala, 2014 & Produção Online \\
\hline Pedro Carlos Resende Junior, 2012 & Revista Brasileira de Gestão de Negócios \\
\hline Rodrigo Eduardo Bampi, 2014 & Produção Online \\
\hline Rogério Tadeu de Oliveira Lacerda, 2014 & Produção Online \\
\hline Sandra Rolim Ensslin, 2014 & Produção Online \\
\hline Tales Andreassi, 2014 & Produção Online \\
\hline Tomás de Aquino Guimarães, 2012 & Revista Brasileira de Gestão de Negócios \\
\hline
\end{tabular}

Fonte: Produção dos próprios autores.

Conforme dados da tabela 1, percebe-se facilmente uma concentração das publicações em apenas dois periódicos. Doze autores realizaram publicação na Revista Brasileira de Gestão de Negócios (RBGN) e dez publicaram na revista Produção Online. Desta forma outra questão norteadora do trabalho também foi atendida, que estava relacionada a identificar em quais periódicos o tema estratégia e/de inovação foi divulgado.

$\mathrm{Na}$ tabela 2, mostram-se os autores e respectivas instituições de origem. Importante ressaltar que a instituição de origem foi assim identificada conforme dados constantes no 
trabalho publicado. Este fato pode causar uma pequena distorção entre a instituição atual e a instituição quando da submissão do trabalho para o periódico.

Outra possível distorção é com relação às informações constantes no trabalho,com relação ao autor e sua efetiva filiação - instituição a qual está vinculado -, pois alguns autores podem informar como instituição de origem, aquelas as quais estão vinculadas em programas de pós-graduação, enquanto alunos desta modalidade, embora funcionalmente possa estar vinculado a outra instituição.

Tabela 2 - Filiação acadêmica e autores

\begin{tabular}{l|l}
\hline \multicolumn{1}{c}{ Filiação Acadêmica } & \multicolumn{1}{c}{ Autores } \\
\hline EAESP/FGV & $\begin{array}{l}\text { Jonas Mendes Constante } \\
\text { Nathalia Fiala } \\
\text { Tales Andreassi }\end{array}$ \\
\hline ESADE & Manuel Francisco Suárez-Barraza \\
\hline Mackenzie & Fábio Luiz ZandovalBonazzi \\
\hline UCS & $\begin{array}{l}\text { Fernanda Lazzari } \\
\text { Gabriel Sperandio Milan } \\
\text { Rodrigo Eduardo Bampi }\end{array}$ \\
\hline UFSC & $\begin{array}{l}\text { Julieta Scheidt Dienstmann } \\
\text { Leonardo Ensslin } \\
\text { Maurício Fernandes Pereira } \\
\text { Rogério Tadeu de Oliveira Lacerda } \\
\text { Sandra Rolim Ensslin }\end{array}$ \\
\hline UFSM & $\begin{array}{l}\text { Eliete Pozzobon Palma } \\
\text { Jordana Marques Kneipp } \\
\text { Luciana Aparecida Barbieri da Rosa }\end{array}$ \\
\hline UNB & $\begin{array}{l}\text { Pedro Carlos Resende Junior } \\
\text { USP }\end{array}$ \\
& $\begin{array}{l}\text { César Augusto Biancolino } \\
\text { ClandiaMaffini Gomes } \\
\text { Emerson AntonioMaccari } \\
\text { Moises Ari Zilber } \\
\text { Tomás de Aquino Guimarães }\end{array}$ \\
\hline
\end{tabular}

Fonte: Produção dos próprios autores.

$\mathrm{Na}$ tabela 2, os autores estão dispostos em ordem alfabética da instituição (sigla) e posteriormente pela ordem alfabética dos autores. Percebe-se que a Universidade de São Paulo (USP) e a Universidade Federal de Santa Catarina (UFSC), apresentam maior número de autores (5). Este fato pode ser justificado pelo fato de ambas as instituições apresentarem há bastante tempo programas de pós-graduação (com mestrado e doutorado) na área de Engenharia de Produção.

Posteriormente aparecem com três autores origem de trabalhos as universidades de Caxias do Sul (UCS), Universidade Federal de Santa Maria (UFSM) e Escola de Administração de Empresas da Fundação Getúlio Vargas (EAESP/FGV). Este fato pode indicar uma relação entre os temas estratégias e/de inovação em trabalhos conjuntos das áreas de Engenharia de Produção e Administração de Empresas, que apresentam programas de pós-graduação em Administração nestas instituições.

Tabela 3 - Autores e respectiva temática e ambiente

\begin{tabular}{c|c|c}
\hline Autor & Temática & Ambiente \\
\hline Pedro Carlos Resende Junior & Inovação. Serviços. Inovação de & Indústrias de \\
\hline
\end{tabular}




\begin{tabular}{|c|c|c|}
\hline Tomás de Aquino Guimarães & serviços & $\begin{array}{l}\text { Engenharia e } \\
\text { Tecnologia e } \\
\text { Hotelaria }\end{array}$ \\
\hline $\begin{array}{l}\text { César Augusto Biancolino } \\
\text { Emerson AntonioMaccari } \\
\text { Maurício Fernandes Pereira }\end{array}$ & $\begin{array}{l}\text { Inovação. Tecnologia da Informação. } \\
\text { Valor do negócio. }\end{array}$ & Empresa de TI \\
\hline Manuel Francisco Suárez-Barraza & $\begin{array}{c}\text { Inovação. Melhoria continua. Processo } \\
\text { de inovação. Gestão pública. Os } \\
\text { governos locais. }\end{array}$ & $\begin{array}{l}\text { Administrações } \\
\text { locais espanholas }\end{array}$ \\
\hline $\begin{array}{c}\text { Fábio Luiz ZandovalBonazzi } \\
\text { Moises Ari Zilber }\end{array}$ & $\begin{array}{l}\text { Inovação. Modelo de negócio. Funil de } \\
\text { inovação. Business ModelCanvas. }\end{array}$ & Empresa DSM \\
\hline $\begin{array}{c}\text { Eliete Pozzobon Palma } \\
\text { ClandiaMaffini Gomes } \\
\text { Jordana Marques Kneipp } \\
\text { Luciana Aparecida Barbieri da Rosa }\end{array}$ & $\begin{array}{l}\text { Estratégias sustentáveis. Desempenho } \\
\text { exportador. }\end{array}$ & $\begin{array}{l}\text { Empresas das gemas } \\
\text { e joias de fabricação. }\end{array}$ \\
\hline $\begin{array}{c}\text { Jonas Mendes Constante } \\
\text { Nathalia Fiala } \\
\text { Tales Andreassi }\end{array}$ & $\begin{array}{l}\text { Spin-Offs. Tecnologia. Inovação. } \\
\text { Empreendedorismo Corporativo. }\end{array}$ & $\begin{array}{l}\text { Pequenas e médias } \\
\text { empresas }\end{array}$ \\
\hline $\begin{array}{l}\text { Julieta Scheidt Dienstmann } \\
\text { Rogério Tadeu de Oliveira Lacerda } \\
\text { Leonardo Ensslin } \\
\text { Sandra Rolim Ensslin }\end{array}$ & $\begin{array}{c}\text { Apoio à Decisão. Desempenho. } \\
\text { Inovação. Revisão Bibliométrica. } \\
\text { Bibliometria. }\end{array}$ & $\begin{array}{l}\text { Pesquisa de artigos já } \\
\text { publicados }\end{array}$ \\
\hline $\begin{array}{l}\text { Fernanda Lazzari } \\
\text { Rodrigo Eduardo Bampi } \\
\text { Gabriel Sperandio Milan }\end{array}$ & $\begin{array}{c}\text { Inovação. Teoria da Vantagem } \\
\text { Comparativa. Indicadores de } \\
\text { Desempenho. Desempenho Financeiro. } \\
\text { Competitividade. }\end{array}$ & $\begin{array}{l}\text { Empresas brasileiras } \\
\text { de capital aberto }\end{array}$ \\
\hline
\end{tabular}

Fonte: Produção dos próprios autores.

Na tabela 3, demonstram-se os ambientes e temáticas dos trabalhos. É possível perceber que não existe uma concentração de trabalhos em determinado ambiente, sendo este os mais diversos. Entretanto chama atenção a ausência de organizações públicas (de governos) nos ambientes. De outra para com relação a temática desperta atenção que o tema "inovação" está presente como temática principal na maior parte dos trabalhos.

\section{Considerações finais}

Por intermédio deste trabalho foi possível apresentar um retrato, com contornos relacionados às questões de pesquisa supramencionadas e que caracterizam os dados levantados, e contribuir na difusão destas informações sobre um estado relativo da produção acadêmica brasileira com relação aos temas propostos.

Neste sentido chama atenção a ausência de organizações públicas nos trabalhos, este fato talvez mereça uma maior investigação dos motivos que fazem com que as organizações públicas 
não sejam objeto de estudos relacionados a estratégia, mas especialmente relacionados a inovação, que é um tema com presença maciça nos trabalhos.

Como sugestão para futuros trabalhos pode-se citar, entre outras perspectivas, a ampliação do espaço temporal de consulta e a abrangência de contemplar bases de dados de outras áreas do conhecimento relacionadas ao tema estratégia e/de inovação, porexemplo a área de Administração (ciências sociais aplicadas) e um levantamento longitudinal em um periódico específico. A produção científica relacionada aos programas de pós-graduação em Engenharia de Produção e/ou áreas afins como a Administração também podem se caracterizar como objeto de pesquisa para outros trabalhos.

Como limitações do trabalho, podemos mencionar o fato de não termos consultado as bases de dados de outras áreas relacionadas ao tema como a Administração e outras áreas de concentração das Engenharias.

\section{Referências}

AMARAL, Roniberto Morato do, POSSATTI, Mario André, FARIA, Leandro I. L.,

ALLIPRANDINI, Dário Henrique, PEREIRA, Neocles Alves. Uma visão da produção científica nos anais do Encontro Nacional de Engenharia de Produção através da bibliometria. In: ENCONTRO NACIONAL DE ENGENHARIA DE PRODUÇÃO, 24, 2004, Florianópolis, SC. Anais do XXIV ENEGEP. Florianópolis, SC: ENEGEP, 2004.

BARBIERI, José Carlos (Org.). Organizações inovadoras: textos e casos brasileiros. Rio de Janeiro: Editora FGV, 2003.

BERTO, Rosa Maria Villares S., NAKANO, Davi Noburu. A produção científica nos anais do Encontro Nacional de Engenharia de Produção: um levantamento de métodos e tipos de pesquisa. Revista Produção, v. 9, n. 2, p. 65-76, 2000.

CALDAS, Miguel Pinto. Análise bibliométrica da produção científica brasileira de RH na década de 90 - um mapeamento a partir das citações dos artigos publicados no Enanpad. São Paulo: FGV EAESP, 2005.

CALLADO, Aldo Leonardo Cunha, ALMEIDA, Moisés Araújo. Perfil dos artigos sobre custos no agronegócio publicados nos anais do Congresso Brasileiro de Custos. Custos e @gronegócioon line, v. 1, n. 1, p. 42-61, jan./jun. 2005.

CALLIGARIS, AlineBelintani et al. Aspectos relevantes da utilização de um programa de inovação tecnológica em pequenas empresas - o PIPE/FAPESP. 2001. In: ENCONTRO NACIONAL DE ENGENHARIA DE PRODUÇÃO, 21, 2001, Salvador, BA. Anais do XXI ENEGEP. Salvador, BA: ENEGEP, 20014.

DUARTE, André Luís de Castro de Moura, PINTO, Áureo Magno Gaspar, OLIVEIRA FILHO, Sebastião Ronaldo de. A produção científica em gestão de operações: uma análise do ENEGEP. In: ENCONTRO NACIONAL DE ENGENHARIA DE PRODUÇÃO, 24, 2004, Florianópolis, SC. Anais do XXIV ENEGEP. Florianópolis, SC: ENEGEP, 2004.

FREEMAN, Christopher, SOETE, Luc. A economia da inovação industrial. Campinas, SP: Editora da UNICAMP, 2008. (Clássicos da Inovação). . 
GIL, A. C. Como elaborar projetos de pesquisa. $4^{\mathrm{a}}$ edição. São Paulo:Atlas; 2002.

GOMES, Giancarlo, MACHADO, Denise Del Prá Netto, GIOTTO, Olivio Tiago. Análise do conteúdo dos artigos de inovação publicados nos anais do ALTEC, SIMPOI e ENANPAD (2003-2007). SIMPOI. Anais. 2009.

HITT, Michael A. IRELAND, Duarte R e HOSKISSON, Robert E. Administração Estratégica. São Paulo: Editora Thomsom. .2007.

HOPPEN, Norberto. Sistemas de Informação no Brasil: uma análise dos artigos científicos dos anos 90. Revista de Administração Contemporânea, v. 2, n. 3, p. 151-177, set./dez. 1998.

HOPPEN, Norberto, MEIRELLES, Fernando S. Sistemas de Informação: um panorama da pesquisa científica entre 1990 e 2003. Revista de Administração de Empresas, v. 45, n. 1, jan./mar. 2005, p. 24-35.

JABBOUR, Charbel José Chiappetta, SANTOS, Fernando César Almada e BARBIERI, José Carlos. Gestão ambiental empresarial: um levantamento da produção científica brasileira divulgada em periódicos da área de Administração entre 1996 e 2005. Revista de Administração Contemporânea, v.12, n. 3, p. 689-715, jul./set. 2008.

KIM, Linsue NELSON, Richard R. (Orgs.).Tecnologia, aprendizado e inovação: as experiências das economias de industrialização recente. Campinas, SP: Editora da UNICAMP, 2005. (Clássicos da Inovação)

LACERDA, Daniel Pacheco, SILVA, Édison Renato Pereira da, NAVARRO, Leonardo Luiz Lima, OLIVEIRA, Níkollas Nunes Pereira e CAULLIRAUX, Heitor Mansur. Algumas caracterizações dos métodos científicos em Engenharia de Produção: uma análise de periódicos nacionais $\mathrm{e}$ internacionais. In: ENCONTRO NACIONAL DE ENGENHARIA DE PRODUÇÃO, 27, 2007, Foz do Iguaçu, PR. Anais do XXVII ENEGEP. Foz do Iguaçu, PR: ENEGEP, 2007.

LOIOLA, Elizabethe BASTOS, AntonioVirgilio Bittencourt. A produção acadêmica sobre aprendizagem organizacional no Brasil. Revista de Administração Contemporânea, v. 7, n. 3, p. 181-201, jul./set. 2003.

MARCANTONIO, Maria Isabel Palmeiro e LAZZERI, Marcia Ines. Indicadores de desempenho em gerenciamento de projeto: um estudo bibliométrico. In: ENCONTRO NACIONAL DA ASSOCIAÇÃO NACIONAL DE PÓS-GRADUAÇÃO EM ADMINISTRAÇÃO, 38, 2014, Rio de Janeiro, RJ. Anais do XXXVIII ENANPAD. Rio de Janeiro, RJ: ENANPAD, 2014.

MIGUEL, P. A. C. (organizador). Metodologia de Pesquisa em Engenharia de Produção e Gestão de Operações. Rio de Janeiro: editora Campus, 2012. 6 edição.

MINISTÉRIO DA CIÊNCIA E TECNOLOGIA. Financiadora de Estudos e Projetos. Manual de Oslo. 3. ed. 2005.

MINTZBERG, H., LAMPE, J.e AHLSTRAND, B. Safári de Estratégia. Porto Alegre; editora Bookman. 2 edição. 2000. 
MOWERY, David C. e ROSENBERG, Nathan. Trajetórias da inovação: a mudança tecnológica nos Estados Unidos da América no século XX. Campinas, SP: Editora da UNICAMP, 2005.

NEELY, Andy e HII, Jasper.Innovation and business performance: a literature review. Universityof Cambridge, 1998.

ROSSONI, Luciano, FERREIRA JÚNIOR, Israel e HOCAYEN-DA-SILVA, Antônio João. Administração de ciência e tecnologia: a produção científica brasileira entre 2000 e 2005. Revista de Administração da UNIMEP, v. 7, n. 3, set./dez. 2009.

SANTOS, Elaine Maria dos, PILATTI, Luiz Albertoe KOVALESKI, João Luiz. Ensino de Engenharia de Produção: análise da produção científica do ENEGEP. In: ENCONTRO NACIONAL DE ENGENHARIA DE PRODUÇÃO, 25, 2005, Porto Alegre, RS. Anais do XXV ENEGEP. Porto Alegre, RS: ENEGEP, 2005.

TINOCO, Tatiana. A produção científica de Administração no Brasil, 1997-2002: uma perspectiva bibliométrica. São Paulo: FGV, 2005. 54 f. Dissertação (Mestrado)- Programa de Pós-Graduação, Escola de Administração de Empresas, Fundação Getúlio Vargas, São Paulo, 2005.

TONELLI, Maria José, CALDAS, Miguel P., LACOMBE, Beatriz Maria Braga e TINOCO, Tatiana. Produção acadêmica em recursos humanos no Brasil: 1991-2000. Revista de Administração de Empresas, v. 43, n. 1, p. 105-122, jan./fev./mar. 2003. 